\title{
Inversion effect of electrohydrodynamic vortices in the isotropic and anisotropic phase of thin nematic layers with two free surfaces
}

\author{
C. Domenici, S. Faetti and L. Fronzoni \\ Istituto di Fisica dell'Università di Pisa, Pisa, Italy and Gruppo Nazionale di Struttura della Materia del CNR, Pisa, Italy
}

(Reçu le 28 octobre 1980, révisé le 1er juin 1981, accepté le 1er juin 1981)

\begin{abstract}
Résumé. - Lorsqu'on applique un champ électrique sur un film mince de cristal liquide nématique ayant deux surfaces libres, on observe plusieurs tourbillons. Dans cet article nous étudions l'effet d'inversion du mouvement qui se produit lorsque on applique une suite d'impulsions de tension.

Nous interprétons cet effet en mécanismes de relaxation du film.

Abstract. - Thin layers of nematic liquid crystal with two free surfaces show a vortex pattern if they are subjected to an electric field. In this paper, we study a characteristic inversion of the hydrodynamic flow, that occurs if a pulsed electric field is applied.

Such an effect is interpreted in terms of the relaxation mechanisms of the layer.
\end{abstract}

An hydrodynamic vortex pattern occurs when an electric field, exceeding a threshold value, is applied parallel to the surfaces of a thin nematic layer having two free surfaces [1-3]. The free layer is obtained by suspending a nematic drop on a rectangular frame made of four wires : two opposite wires are conducting, whilst the others are insulating. The electric voltage is applied between the conducting wires (long sides of the rectangle) in such a way to generate an electric field parallel to the free surfaces. The vortex pattern consists of a periodical series of adjacent vortices with average vorticity perpendicular to the free surfaces (Fig. 1c). The vortex mode (V.M.) occurs both in the anisotropic (Fig. 1e) and isotropic phase (Fig. 1f), without important variations near the isotropic-anisotropic transition temperature. Therefore the V.M. cannot be interpreted in terms of the anisotropy of the nematic [4]. In a previous paper [3] we have shown that also the charge injection from electrodes [5] cannot explain our experimental results.

In this paper we study a peculiar «inversion effect » that occurs when a sequence of voltage pulses is applied to the nematic layer. This effect can be interpreted in terms of the relaxation mechanisms of electric space charges and of the hydrodynamic relaxation of the layer.

1. Experiment. - All our measurements were performed on homeotropic free layers of MBBA [6]. The temperature of the layers was held fixed within $\pm 0.1{ }^{\circ} \mathrm{C}$. The optical observations were made by using a polarizing microscope in the transmission or reflection mode. The shapes of the voltage pulses used in our experiment are shown in figures $2 a$ and $2 b$. The repetition time $T_{0}$ and the length $T_{0}^{\prime}$ of the voltage pulses could be changed independently from $10^{-3}$ to $10 \mathrm{~s}$, whilst the pulse amplitude $A$ could be changed between 1 and $800 \mathrm{~V}$. Most of our experiments were performed with $T_{0}^{\prime} \sim 1 \mathrm{~s}$ in such a way to have a stationary vortex motion before removing the electric field.

Figure $1 a$ and figure $1 e$ show the vortex pattern when the layer is observed between crossed polarizers or whithout polarizers, respectively. The pattern in figure $1 a$ (crossed polarizers) is due to the tilt of the director induced by the hydrodynamic flow. The pattern in figure $1 e$ (without polarizers) is caused by two simultaneous effects : a) the spatial variation of the refractive index due to the nonuniform orientation of the director ; $b$ ) the nonuniformity of the thickness of the layer produced by the hydrodynamic flow. Both these effects generate a diffraction of the transmitted light. We remark that the latter mechanism does not depend on the nematic anisotropy, and in fact we also observe the image of the V.M. in isotropic phase (Fig. 1f).

The vortex mode can occur in two different configurations (Figs. $1 a$ and $1 b$ ) independently on the sign of the applied electric field. By observing the motion of small particles suspended on the free layer we find 


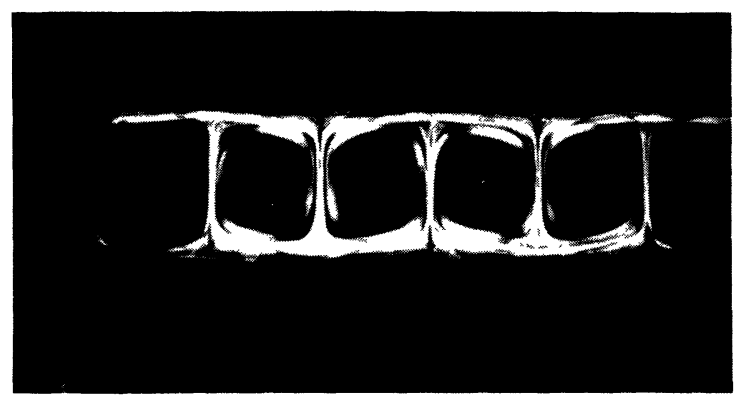

(a)

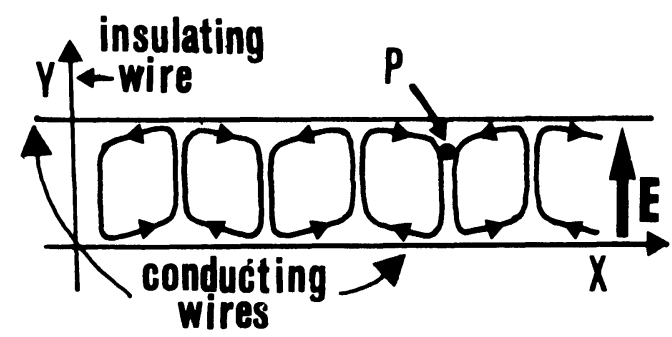

(c)

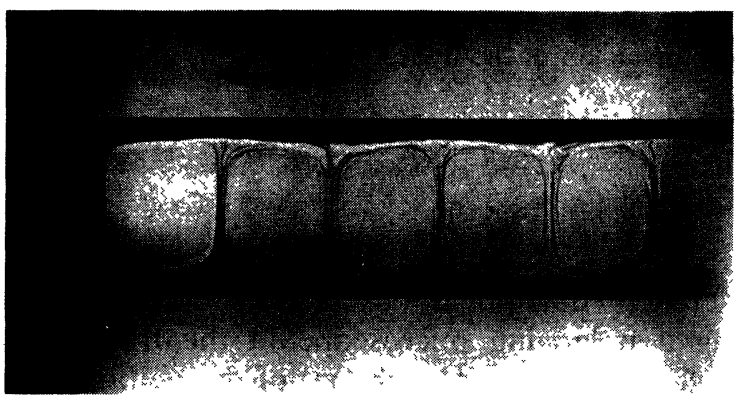

(e)

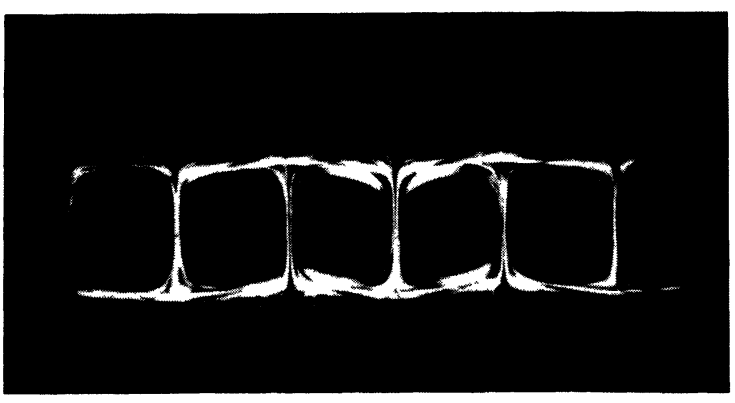

(b)

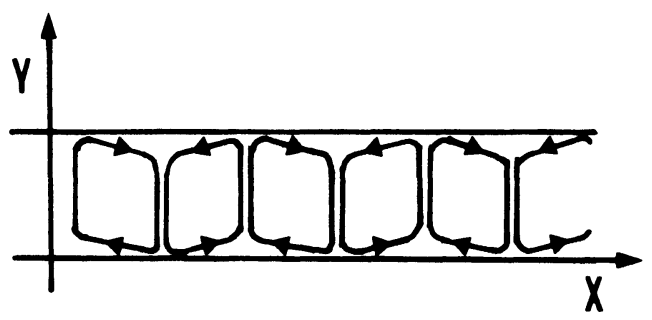

(d)

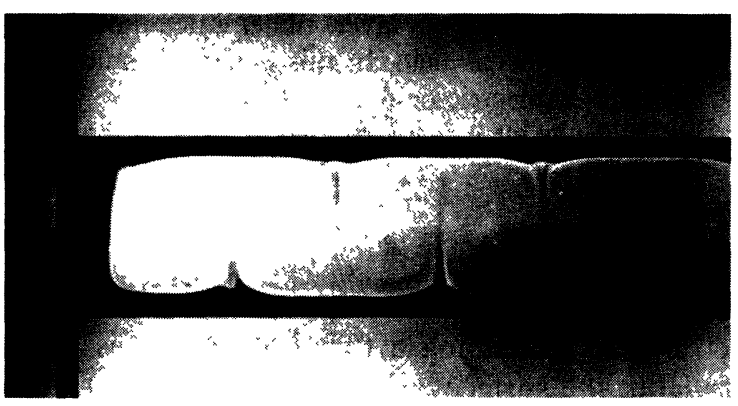

(f)

Fig. 1. $-a$ ) and $b$ ) Vortex pattern configurations between crossed polarizers. $c$ ) and $d$ ) Flow lines corresponding to the vortex patterns in figures $1 a$ and $1 b$ respectively. $e$ ) Vortex pattern observed without polarizers in anisotropic phase. (Temperature $T=30^{\circ} \mathrm{C}$.) $f$ ) Vortex $^{\circ}$ pattern observed without polarizers in isotropic phase. (Temperature $T=50^{\circ} \mathrm{C}$.)
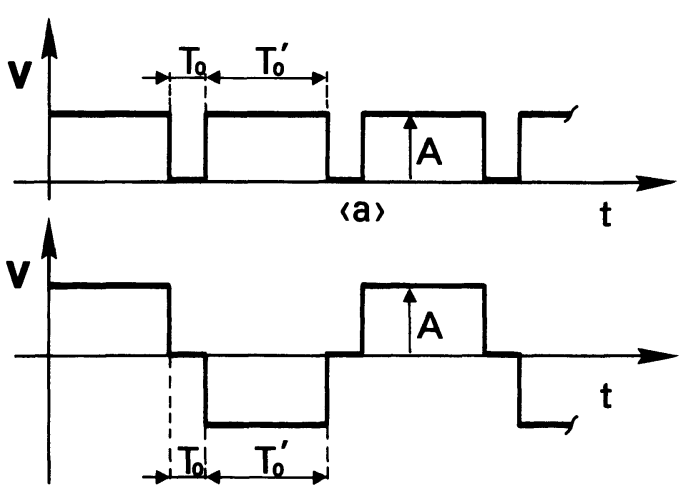

(b)

Fig. 2. - a) Waveform with same polarity pulses. b) Waveform with opposite polarity pulses.

different flow lines corresponding to the configurations in figures $1 a$ and $1 b$.

These flow lines are schematically drown in figures $1 c$ and $1 d$. Let $x y z$ an orthogonal right-hand coordinate system with $x$-axis parallel to the conducting wires and $y$-axis parallel to the insulating wires (Fig. 1c). We define as $\omega(i)=\left\langle(\operatorname{rot} v)_{z}\right\rangle$ the average value of the $z$-component of the vorticosity within the $i$-th vortex, from one insulating wire. We notice that the patterns in figures $1 a$ and $1 b$ correspond to opposite values of $\omega(i)$.

2. Flow inversion effect. - A sequence of voltage pulses with the same polarity (see Fig. $2 a$ ) is applied between the conducting wires. By changing the pulse repetition time $T_{0}$, we find three different regimes. The transition between contiguous regimes occurs in correspondence of two characteristic repetition times $T_{\mathrm{i}}$ and $T_{\mathrm{R}}$. Let's indicate with $\omega(i, j)$ the stationary value of $\omega(i)$ during the $j$-th voltage pulse. Changing $T_{0}$ we find :

i) For $T_{0}<T_{\mathrm{i}}$, the vortex patterns observed during two successive pulses are identical and, so,

$$
\omega(i, j+1)=\omega(i, j) .
$$


ii) For $T_{\mathrm{i}} \leqslant T_{0}<T_{\mathrm{R}}$, the patterns in figures $1 a$ and $1 b$ appear alternatively during successive pulses. Therefore $\omega(i, j+1)=-\omega(i, j)($ flow inversion effect $)$. We name $T_{\mathrm{i}}$ as the " inversion time".

iii) For $T_{0}>T_{\mathrm{R}}$ (random time), the patterns in figures $1 a$ and $1 b$ occur with the same statistical probability. Therefore $\omega(i, j+1)= \pm \omega(i, j)$.

If we apply a sequence of pulses with alternate polarities (Fig. $2 b$ ), we find that the flow inversion occurs always if $0 \leqslant T_{0}<T_{R}^{*}$ where $T_{R}^{*} \sim T_{R}$ within the experimental accuracy. If $T_{0} \geqslant T_{\mathrm{R}}^{*}$, we observe the random behaviour of the point iii).

The times $T_{\mathrm{i}}, T_{\mathrm{R}}, T_{\mathrm{R}}^{*}$ depend on the temperature and on the pulse amplitude. Figure 3 shows the dependence of $T_{\mathrm{i}}$ on the pulse amplitude, whilst figure 4 shows the dependence of $T_{\mathrm{i}}$ on the temperature. We

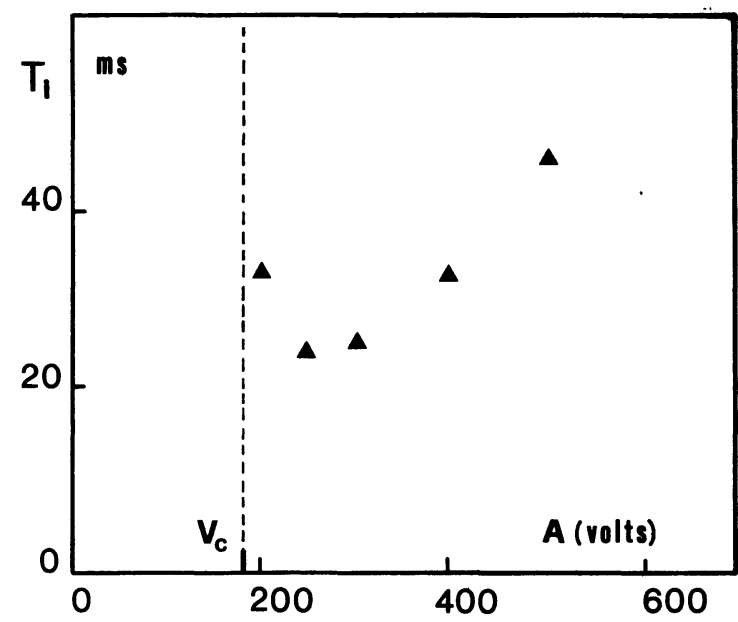

Fig. 3. - Inversion time $T_{\mathrm{i}}$ versus the pulse amplitude $A . V_{\mathrm{c}}$ is the threshold voltage $(180 \mathrm{~V})$ of the nematic free layer. (Temperature $T=22^{\circ} \mathrm{C}$.)

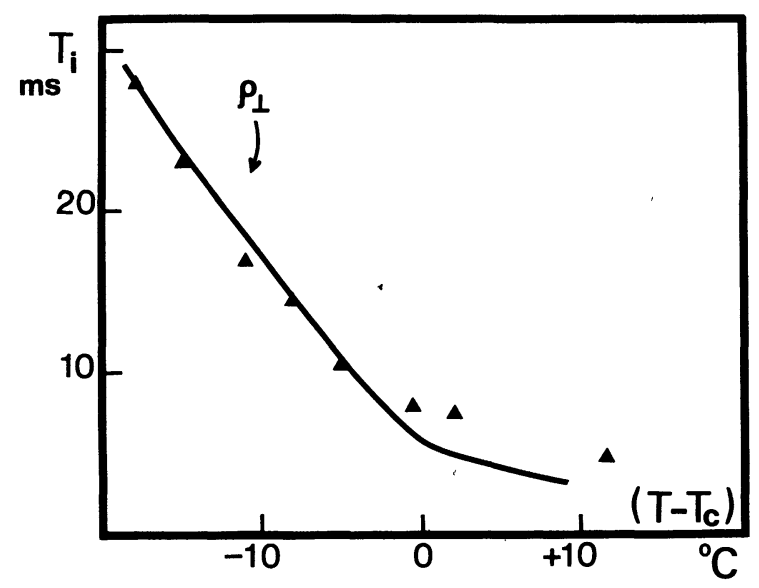

Fig. 4. - Inversion time $T_{\mathrm{i}}$ versus $T-T_{\mathrm{c}}$, where $T$ is the temperature and $T_{\mathrm{c}}=41^{\circ} \mathrm{C}$ is the isotropic-anisotropic phase transition temperature. The full line shows the temperature dependence of the component of the resistivity tensor perpendicular to the optical axis (director) in arbitrary unities. $\rho_{\perp}$ is deduced from reference [9] (Threshold voltage $V_{\mathrm{c}}=180 \mathrm{~V}$, pulse amplitude $A=400 \mathrm{~V}$.) remark that $T_{\mathrm{i}}$ decreases with the temperature without any sharp variation near the isotropic-anisotropic fluid transition temperature $T_{\mathrm{c}}$. Therefore the inversion effect seems to be largely independent on the anisotropy of the nematic liquid crystal. The experimental values of $T_{\mathrm{R}}$ and $T_{\mathrm{R}}^{*}$ at room temperature are typically of the order of some seconds. The temperature dependence of $T_{\mathrm{R}}$ and $T_{\mathrm{R}}^{*}$ is qualitatively similar to the one of the inversion time $T_{\mathrm{i}}$.

In order to understand the inversion effect we have observed the relaxation of the system after removing the electric field. The image between crossed polarizers shows that the layer restores its homeotropic alignment after a small time $\tau$. For example we have found $\tau \simeq 8 \mathrm{~ms}$ for a layer with average thickness $\bar{h}=3 \mu \mathrm{m}$ at room temperature. Since the tilt of the director is induced by the viscous torque, also the vorticity has already relaxed within the time $\tau$. We notice that the measured value of $\tau$ is of the order of the typical expected values of the director relaxation time $\tau_{d}$ [7] and of the vorticity relaxation time $\tau_{\mathrm{v}}$ [8]. In fact

$$
\tau_{\mathrm{d}} \sim \frac{\gamma_{1} \bar{h}^{2}}{\pi^{2} k}=10 \mathrm{~ms} \quad \text { and } \quad \tau_{\mathrm{v}} \sim \frac{d^{2}}{\pi^{2} v}=3 \mathrm{~ms},
$$

where $\gamma_{1}$ is a viscosity coefficient $(\simeq 0.7$ poise), $k$ is an average elastic constant $\left(\simeq 0.6 \times 10^{-7}\right), \bar{h}$ is the average thickness of the layer $(=3 \mu \mathrm{m}), d$ is the distance between the conducting wires $(=0.5 \mathrm{~mm})$ and $v$ is an average cinematic viscosity coefficient $(\simeq 0.2$ poise). If we observe the V.M. without polarizers (Fig. 1e), we find that the vortex pattern relaxes very slowly with times of the order of some seconds. We remind that the pattern observed without polarizers is due to the tilt of the director and to the nonuniformity of the local thickness of the layer. The director relaxes in a short time $(\sim \mathrm{ms})$, therefore the slow relaxation must be attributed to the relaxation of the film shape. This interpretation is confirmed by the interferometric measure of the local thickness of the layer. A slow hydrodynamic motion is associated with the layer relaxation both in isotropic and anisotropic phase. The local vorticity of such a slow motion is opposite and about thousand times lower than the one of the previous vortex pattern.

This inverted motion (backflow), can be explained in terms of the thickness variations induced by the vortex motion. In absence of vortices the profile of thickness $h(x, y)$ is almost uniform (within $0.5 \mu \mathrm{m}$ ) in a large central region of the layer, whilst it changes greatly in a narrow stripe ( $\sim 50 \mu \mathrm{m}$ width) near the wires. Because of the symmetry of the layer

$$
\left.\frac{\partial h}{\partial y}\right|_{y}=-\left.\frac{\partial h}{\partial y}\right|_{d-y}<0
$$

where $y=0$ and $y=d$ are the $y$-coordinates of the conducting wires. 
In presence of a hydrodynamic velocity $\mathbf{v}$, the local thickness of the incompressible nematic changes as

$$
\left(\frac{\partial h}{\partial t}\right)=-h\left(\frac{\partial \bar{v}_{x}}{\partial x}+\frac{\partial \bar{v}_{y}}{\partial y}\right)-\bar{v}_{x} \frac{\partial h}{\partial x}-\bar{v}_{y} \frac{\partial h}{\partial y}
$$

where $\bar{v}_{x}$ and $\bar{v}_{y}$ are the $x-$ and $y$-components of the average value of the hydrodynamic velocity along the $z$ vertical axis.

Because of the nonuniformity of $h$ along the $y$ axis, the term $-v_{y} \frac{\partial h}{\partial y}$ generates a variation of the local thickness untill a stationary state is reached. This convective term produces an increase of the thickness in points where $v_{y}$ and $\partial h / \partial y$ have opposite signs (point $P$ in figure $1 c$ ). In fact the liquid is dragged toward region $\mathbf{P}$ from the adjacent and thicker region near the conducting wire $(y=d)$. When the electric field is removed, some liquid leaves region $\mathrm{P}$ toward the adjacent region near the conducting wire, because of the elastic return forces due to the surface tension. This motion corresponds to the observed backflow.

3. Discussion. - The main features of the flow inversion effect can be explained in terms of the relaxation of the system. Before discussing this relaxation we recall, for convenience, the qualitative mechanism of onset of the instability according with the " surface charge model " that we have recently proposed [3]. Since the vortex mode is largely independent of the anisotropy of the nematic [3], we neglect such an anisotropy in our analysis.

When a d.c. voltage $V=V(0)-V(d)$ is applied between the conducting wires, some electric charges are accumulated on the two free surfaces because of the discontinuity of the electric conductivity $\sigma$ at the nematic-air interfaces. At the equilibrium the surface electric charge density far from the insulating wires is

$$
q_{0}(y)=\alpha(y) \frac{V}{d}
$$

where $\alpha(y)$ is an adimensional positive function that depends on the geometric shape of the layer. $\alpha(y)$ decreases as $y$ increases $(\partial \alpha / \partial y<0)$. The maximum value of $\alpha(y)$ is near the positive electrode and is of the order of the unity. The surface charge arrangement is stable only if $V$ is lower than a threshold voltage $V_{\mathrm{c}}$. If $V>V_{\mathrm{c}}, q_{0}(y)$ becomes unstable with respect to small electric or hydrodynamic fluctuations. Let's consider, for instance, a vortex fluctuation with $y$-component of the velocity $v_{y}=v \cos k x$. This vortex motion generates a new surface charge arrangement $q_{0}+\delta q$ because of the convective term

$$
\left(\frac{\partial \delta q}{\partial t}\right)=-\frac{\partial q_{0}}{\partial y} v \cos k x \text {. }
$$

Since $\partial \alpha / \partial y<0$, the new electric surface force is directed in such a way to sustain the initial vortex fluctuation. If $V>V_{\mathrm{c}}=B \sqrt{\eta \sigma}$, the integral of the electric force along a closed flow line exceeds the viscous one and the vortex velocity increases untill it reaches a stationary value. $B$ is a coefficient that depends on the shape of the layer and on the dielectric coefficients; $\eta$ and $\sigma$ are viscosity and electric conductivity coefficients respectively.

The qualitative features of the vortex instability in pulsed electric fields can be explained in terms of the previous mechanisms and of the relaxation times of the surface charges $\left(\tau_{\text {charge }}\right)$ and of the backflow $\left(\tau_{\text {back }}\right)$. The average relaxation time of the surface charges after removing the electric field is

$$
\tau_{\text {charge }}=\frac{1}{4 \pi \sigma_{\perp}} f
$$

where $\sigma_{\perp}$ is the conductivity coefficient perpendicular to the optical axis (director), whilst $f$ is an adimensional function of the dielectric and electric conductivity tensors; it depends on the shape of the layer and on the surface charge profile $q(x, y)$.

In our experimental conditions the surface charge arrangement and the shape of the layer are very complicated, so we are not able to give the analytical expression of $f$. However, in order to evaluate a qualitative behaviour of the function $f$ we consider a thin homeotropic plane layer with thickness $h$ and surface charge density $q=A \cos k x$ on the two free surfaces. If $k h \ll 1$ we can easily show that, after a fast transient, the surface charge relaxes exponentially with the time constant

$$
\tau=\frac{1}{2 \pi \sigma_{\perp} k h}
$$

In such a case, assuming the full relaxation occurs in a time $\tau_{\text {charge }}$ of the order of $3 \tau$, we find $f=6 / k h$. Since the thickness of the layer is typically $2 \mu \mathrm{m}$ in the centre of the layer and $20 \mu \mathrm{m}$ near the conducting wires, and $k \simeq 10^{2} \mathrm{~cm}^{-1}$, we estimate from equation (6) $f \simeq 30 \div 300$. In our MBBA sample (not purified) $1 / 4 \pi \sigma_{\perp}=0.5 \mathrm{~ms}$, so $\tau_{\text {charge }} \simeq 15 \div 150 \mathrm{~ms}$.

Changing the repetition time $T_{0}$ between two successive pulses $j$ and $j+1$, we distinguish three different cases :

1) $T_{0}>\tau_{\text {back }}>\tau_{\text {charge }}$. In this case the system has completely relaxed when the $(j+1)$-pulse occurs. Therefore the sign of $\omega(i, j+1)$ is only determined by the random fluctuation that triggers the instability. This random behaviour is found experimentally when $T_{0}>T_{\mathrm{R}}$, so we can assume $T_{\mathrm{R}} \simeq \tau_{\text {back}}$.

2) $\tau_{\text {charge }}<T_{0}<\tau_{\text {back. }}$. In this case the slow backflow is still on when the $(j+1)$-electric pulse occurs. Such a motion plays the same role of the hydrodynamic fluctuation that triggers the vortex instability. Therefore $\omega(i, j+1)=-\omega(i, j)$, since the local 
vorticity of the backflow is opposite to the one of the previous vortex pattern.

3) $T_{0}<\tau_{\text {charge }}$. In this case both the periodic surface charge $q$ and the shape of the layer have not relaxed when the $(j+1)$-electric pulse occurs. Therefore a periodic electric surface force $q E_{j+1}$ is applied on the layer as soon as the $(j+1)$-electric pulse occurs. If $E_{j+1}=-E_{j}$, the surface force is locally inverted with respect to the one during the previous pulse, so $\omega(i, j+1)=-\omega(i, j)$. If $E_{j+1}=E_{j}$, the surface force has locally the same versus of the one during the previous pulse, so $\omega(i, j+1)=\omega(i, j)$. However we must remark that, in this case, the effects due to the residual surface charges and to the backflow are conflicting. If the repetition time $T_{0}$ is close to $\tau_{\text {charge }}$, the residual charge is very small and the inverting effect of the backflow can become dominant. Therefore the measured inversion time $T_{\mathrm{i}}$ should be of the order of $\tau_{\text {charge }}$. Since $\tau_{\text {charge }}$ is proportional to the resistivity coefficient $\rho_{\perp}=\frac{1}{\sigma_{\perp}}$, we expect also $T_{\mathrm{i}} \propto \rho_{\perp}$. This behaviour agrees qualitatively with the temperature dependence of $T_{\mathrm{i}}$ (Fig. 4).

However a more detailed theoretical analysis is necessary in order to explain all the experimental observations. This analysis is now in progress in our laboratory.

\section{References}

[1] Meyerhofer, D., Sussman, A., Williams, R., J. Appl. Phys. 43 (1972) 3685.

[2] Faetti, S., Fronzoni, L., Rolla, P. A., Stoppini, G., Lett. Nuovo Cimento 17 (1976) 475.

[3] Faetti, S., Fronzoni, L., Rolla, P. A., J. Physique Colloq. 40 (1979) C3-497.

[4] Helfrich, W., J. Chem. Phys. 51 (1969) 4092.
[5] Felici, N., Rev. Gen. Electr. Sci. 76 (1967) 786.

[6] Faetti, S., Fronzoni, L., Solid State Commun. 25 (1978) 1087.

[7] Pieranski, P., Brochard, F., Guyon, G., J. Physique 34 (1973) 35.

[8] TAYLOR, G. I., Philos. Mag. (6) 46 (1923) 671.

[9] Rondelez, F., Diguets, D., Durand, G., Mol. Cryst. Liq. Cryst. 15 (1971) 183. 